\title{
Feasibility of a Practical Clinical Trial for Asthma Conducted in Primary Care
}

\author{
David L. Hahn, MD, MS, and Mary Beth Plane, PhD
}

Background: Practical clinical trials (PCTs) are essential to generate relevant evidence-based information to improve patient health. Primary care physicians' experience performing randomized controlled trials (RCTs) on representative patient populations is limited. We implemented a pilot practice-based asthma PCT to answer the following feasibility questions: (1) Was clinician interest initiated and maintained? (2) Did clinicians enroll patients into an RCT and complete follow-up? (3) Was an interactive voice-response (IVR) telephone system useful to collect patient-reported data?

Methods: The protocol included (1) broadly representative adult asthma eligibility criteria, (2) selfreported patient-oriented outcomes, and (3) use of IVR to collect these data. Physicians in practicebased research networks, managed care organizations, and academic networks volunteered to participate.

Results: Of 13 physician volunteers, 10 (8 single-person office practices, 1 emergency department physician, 1 clinical researcher) from 4 states and 1 Canadian province enrolled 58 subjects and randomized 45 meeting final eligibility criteria; 39 (87\%) attended the follow-up visit. However, only 34 (76\%) provided adequate follow-up IVR self-report data, and subjects with less than a high school education provided significantly $(P<.001)$ less data than other groups.

Conclusions: Physician recruiting, randomizing, and completing a representative sample of adult asthma patients was feasible. The utility of IVR in primary care research requires further study. (J Am Board Fam Pract 2004;17:190 -5.)

The growing practice-based research (PBR) movement has made significant contributions to current understanding of the content, process, and outcomes of primary care medical practice in North

Submitted, revised, 2 December 2003.

From the Department of Family Practice, Dean Medical Center, Madison, Wisconsin (DLH); Department of Family Medicine, University of Wisconsin Medical School, Madison, Wisconsin (DLH, MBP). Address correspondence to Dr. D. L. Hahn, East Clinic, 1821 S. Stoughton Road, Madison, WI 53716 (e-mail: dlhahn@wisc.edu).

This work was presented in part at the 2002 American Academy of Family Physicians (AAFP) Convocation of Practices, Kansas City, MO, and at the 2002 Wisconsin Primary Care Research Forum, Wisconsin Dells, WI.

The American Academy of Family Physicians/Foundation (AAFP/F) Joint Grant Awards Program provided funds to defray clinical site costs; the North American Primary Care Research Group (NAPCRG) Pfizer Investigator-InPractice Award provided investigator support; the Wisconsin Academy of Family Physicians (WAFP) under the auspices of the Wisconsin Research Network (WReN) supported the Clinical Coordinating Center; the Dean Foundation for Health Research and Education defrayed the costs of recruitment at one site; Pfizer, Inc. provided drug/ placebo and an unrestricted educational grant that was used for the interactive voice-response (IVR) technology. Pfizer, Inc. had no role in study design, conduct, analysis, manuscript preparation or approval. DLH has received speaker honoraria and an unrestricted educational grant from Pfizer, Inc.
America. ${ }^{1,2}$ PBR research methodologies have included descriptive (eg, card studies) and analytic (case-control or cohort studies) approaches. Trials randomized at the clinic, ${ }^{3,4}$ physician, ${ }^{5}$ and patient ${ }^{6}$ levels have contributed important information on management of chronic diseases (eg, depression, ${ }^{4,5}$ problem drinking, ${ }^{6}$ and cardiovascular risk reduction $\left.^{3}\right)$. A few academic networks have performed pharmaceutical contract research. ${ }^{7}$ Experience performing investigator-initiated randomized, controlled trials (RCTs) of medication treatments in primary care practice is limited. ${ }^{8,9}$

Clearly, practice-level interventions such as those described above must be performed in primary care settings. It is less obvious whether primary care-based research is required or preferred when the patient rather than the practice is the unit of analysis. A requirement favoring primary carebased patient-level RCTs is the need for a representative population with a common condition that first presents in primary care and that is rarely referred to specialists or academic centers. Adult asthma is a condition that is commonly encountered in primary care ${ }^{10,11}$ that is rarely referred to specialists, ${ }^{12}$ and for which there exists an inade- 
quate knowledge base $\mathrm{e}^{13}$ on which to formulate evidence-based guidelines. ${ }^{14}$

Short-term drug efficacy studies (of biologic effects in highly selected patients) are overrepresented in current asthma research. ${ }^{13}$ Practical clinical trials (PCTs) (of clinically relevant interventions in representative patient populations) are needed to close widespread gaps in evidence-based knowledge. ${ }^{15}$ We designed and tested the feasibility of a practice-based PCT of adult asthma performed by primary care physicians. In this article, we address 3 primary feasibility questions: (1) Was clinician interest initiated and maintained? (2) Did clinicians successfully enroll patients into an RCT and complete follow-up? (3) Was an interactive voice-response (IVR) telephone system useful to collect patient-reported data?

\section{Methods}

\section{Recruitment of Physicians}

The principal investigator recruited a convenience sample of practicing physicians during meetings of the Ambulatory Sentinel Practice Network (ASPN), the North American Primary Care Research Group (NAPCRG), and the Wisconsin Research Network (WReN). An asthma specialist practicing at a community-based managed care organization (MCO) and an emergency department physician at Cook County Hospital, Chicago, IL, also participated. A list of co-investigators is presented in the Acknowledgments. A target enrollment goal of 6 patients per practice was chosen by a focus group of practicing physicians attending a WReN Annual Meeting.

\section{Recruitment of Patients}

Practicing physicians identified a convenience sample of potentially eligible subjects during the course of usual primary care practice. Four practices with infrastructure support to perform practice-based research provided the assistance of a trained research associate (RA). The remaining physicians recruited patients and collected data themselves. One inner-city emergency department physician identified subjects presenting to the emergency department. Research assistants at the MCO asthma research center recruited subjects through advertisements. Eligible patients met accepted criteria for a diagnosis of stable, persistent asthma confirmed by pulmonary function evidence for revers- ible airways obstruction. ${ }^{16,17}$ Smoking, co-existing fixed obstruction and guideline therapy adherence were not exclusion or inclusion criteria.

\section{Study Protocol}

The clinical question was "Does azithromycin confer lasting benefit to adults bothered by asthma?" The study design was a randomized, triple-blinded (investigator, subject, data analyst), placebo-controlled, parallel group trial of azithromycin as adjunctive treatment for adults with stable, persistent asthma. Eligible patients received study drug or placebo for 6 weeks and attended the follow-up visit 3 months after finishing treatment. Patients used IVR to report asthma symptom and activity scales, amount of rescue bronchodilator administered, and AM and PM peak expiratory flow rate for 1 24-hour period weekly. Patients also used IVR at randomization and at follow up to complete asthma-specific and generic quality-of-life instruments. Patients received no compensation for participation. The protocol was reviewed and approved by the human subjects committees at each participating site. Before enrollment, all subjects provided written informed consent.

\section{Study Management}

Researchers provided each site with a written comprehensive training manual and held periodic telephone conferences during the planning, site initiation, and follow-up phases. The sites faxed data forms to the central site and completed communications involving data issues primarily by e-mail and occasionally by telephone. A research associate dispensed blinded study medication and completed data collection at 4 practices and the MCO site. A trained pharmacist dispensed study medication at the ER site. The participating physician dispensed medication and collected baseline and follow up data at the other sites.

\section{Patient Exit Interview}

Patients completed an exit interview on whether study instructions were easy to understand, whether participation was convenient, whether they liked the IVR system or would have preferred another way of reporting symptoms, and whether their understanding of asthma improved as a result of study participation. After some subjects had completed the study, 3 questions were added (whether they would have been willing to provide 


\begin{tabular}{lccc}
\hline Site descriptions & $\begin{array}{c}\text { No. Subjects } \\
\text { Randomized }\end{array}$ & $\begin{array}{c}\text { No. Months to } \\
\text { Recruit Subjects }\end{array}$ & $\begin{array}{c}\text { No. Subjects Randomized } \\
\text { per 6-Month Interval }\end{array}$ \\
\hline $\begin{array}{l}\text { Single physician practices* } \\
1\end{array}$ & 8 & 18 & 2.7 \\
2 & 4 & 9 & 2.7 \\
3 & 5 & 11 & 2.7 \\
4 & 3 & 11 & 1.6 \\
5 & 2 & 6 & 2.0 \\
6 & 4 & 7 & 3.4 \\
7 & 4 & 7 & 3.4 \\
8 & 5 & 6 & 5.0 \\
AR $^{\dagger}$ & & & 2.9 \\
$\mathrm{MCO}^{\ddagger}$ & 3 & 2 & 7.2 \\
\hline
\end{tabular}

* Eight single-physician primary care practices, of which numbers 2, 3, 5, and 8 were $50 \%$ full-time academic practices. Sites 3 to 6 were assisted by research associates.

${ }^{\dagger}$ A participating physician recruited from an inner-city emergency room.

${ }^{\ddagger}$ Research assistants recruited from a managed care organization (MCO) asthma research center.

data for an entire year, how many days per week they would have been willing to use the IVR system, and if they would be willing to be contacted later about their asthma). Respondents had the opportunity to answer open-ended questions about what they liked best and least about the study.

\section{Statistics and Qualitative Data}

Analysis of variance was used to analyze the loss to follow-up rate and the number of IVR calls by educational level. The $\chi^{2}$ test was used to compare categorical data and an unpaired $t$ test was used to compare continuous data between enrolled patients who were randomized or not. Patient exit interview data were tabulated and described. The principal investigator (DLH) also collected qualitative data from site physicians during site visits and telephone conversations.

\section{Results}

\section{Was Clinician Interest Initiated and Maintained?}

Thirteen physicians volunteered initially to enroll subjects: 11 primary care practitioners (10 family physicians and 1 internist), 1 emergency department physician, and 1 community-based pediatric pulmonologist/allergist affiliated with an asthma research center. Of 11 primary care practitioners, 3 failed to enroll subjects. One private practice physician with no prior experience in clinical research decided that the protocol was too difficult. Another private practitioner with extensive experience in PBR invited patients but none agreed to enroll. An academic physician with a limited practice was unable to enroll subjects. All 3 physicians indicated that they ceased participation because of practical issues, not because they became uninterested.

\section{Did Clinicians Enroll Patients into an RCT and Complete Follow-up?}

Of 58 consenting subjects, 45 completed the run-in period, met final eligibility criteria, and became randomized. The majority of the 58 enrollees had never been skin-tested, and $38 \%$ met pulmonary function criteria for reversible airway obstruction because peak expiratory flow rates had been recorded but the patients had not had spirometry. Almost half (43\%) reported that their asthma first became symptomatic after an acute respiratory illness. The median age of onset of initial symptoms leading to an asthma diagnosis was 31.5 years. Fifty-one percent of enrollees had mild persistent asthma and $46 \%$ had moderate persistent asthma. There were no significant differences between randomized $(\mathrm{n}=45)$ and nonrandomized $(\mathrm{n}=13)$ subjects for any of these characteristics.

Of the 45 randomized subjects, 39 (87\%) attended the follow-up visit. Attendance at follow-up was directly associated with education (12 of 12 with a bachelor's degree, 25 of 28 completing high school, and 2 of 5 with less than a high school 


\begin{tabular}{lccc}
\hline & $\begin{array}{c}\text { Agree/ } \\
\text { Strongly Agree }\end{array}$ & Neutral & $\begin{array}{c}\text { Disagree/ } \\
\text { Strongly Disagree }\end{array}$ \\
\hline Study instructions were easy to understand. & 25 & 1 & 0 \\
It was convenient for me to participate. & 22 & 3 & 1 \\
I liked using the telephone response system. & 20 & 4 & 2 \\
I would prefer another way of reporting symptoms. & 3 & 11 & 4 \\
My understanding of my asthma has improved as a & 11 & 6 \\
$\quad$ result of participating in this study. & & & 17 \\
\hline
\end{tabular}

education, $P<.01$ ). Table 1 presents recruiting data as time in months required to enroll and randomize patients for the 10 individual sites. The 8 primary care providers randomized an average of 4.4 subjects over 9.4 months, equivalent to an average of 2.9 subjects per practitioner in a 6-month time period. Because 4 physicians did not work full time, the rate per full-time practice somewhat exceeds 3 patients in 6 months. The MCO clinical research site recruited twice that number of subjects in the same period.

\section{Was an IVR Telephone System Useful to Collect Patient-Reported Data?}

The typical weekly IVR telephone call took 4 to 5 minutes. The longer entry and exit quality-of-life call typically took 15 to 25 minutes. For the 39 randomized subjects who attended the follow-up visit, utilization of IVR was significantly $(P<.001)$ related to education: college-educated subjects averaged 19.8 (S.D. 7.6) calls, high-school educated subjects averaged 18 (S.D. 5.7) calls, and subjects without a high school diploma averaged 6 (S.D. 1.4) calls. Subjects $(n=15)$ with 1 or more emergency visits or hospitalizations for asthma in the previous 2 years made fewer IVR calls (mean 14.6, S.D. 7.6) than those $(\mathrm{n}=24)$ without this measure of severity (20.1, S.D. 5.4) $(P=.02)$. Thus, patients with less education and higher utilization of health services for poor asthma control were less likely than others to provide a complete IVR data set, although they did return to the clinical site for study visits.

\section{Patient Exit Interview}

Twenty-six (67\%) of 39 subjects attending the follow up visit returned the patient exit questionnaire and 20 of these questionnaires contained the supplemental questions. Responses to the initial questions on the patient exit questionnaire are pre- sented in Table 2 and show an overall positive response to using the IVR system. Of the $20 \mathrm{pa}-$ tients who responded to the supplemental questions, $18(90 \%)$ answered that they would have been willing to participate for 1 year and would be willing to be contacted later. Only 4 of 18 (22\%) responded that they would be willing to use the IVR system more than once per week.

\section{Discussion}

This pilot study addressed important feasibility questions regarding both the ability of primary care physicians to conduct a practical clinical trial of asthma and the utility of an IVR system to collect patient-reported data. Among physician volunteers, inability to participate was caused by practical considerations (lack of time, experience, patient factors, etc) rather than by lack of interest, suggesting that it will not be difficult to enroll primary care clinicians in adequately funded future studies. On average, each volunteer randomized 3 adults with asthma in a 6-month period. These busy practicing physicians probably overlooked other asthma patients who would have been eligible. In future studies, the per-practice recruitment rate could probably be increased using systematic case identification. Supported by minimal resources and training, the volunteers had a follow-up rate of more than $85 \%$ of randomized subjects, suggesting that an ongoing provider-patient relationship promoted completion of study visits. Perhaps patient incentives could even further improve this excellent follow-up rate.

The need for practical clinical trials on representative patient populations has recently been emphasized. ${ }^{15}$ Management of asthma patients enrolled in this study deviated from the "ideal" profile depicted in expert guidelines. ${ }^{16}$ The majority of enrolled subjects had never been skin-tested, 22\% 
were not on guideline therapy, and $38 \%$ had not had prestudy spirometry. Furthermore, $43 \%$ of them reported the "infectious asthma syndrome" (asthma beginning after an acute lower respiratory tract illness), and the majority had adult-onset asthma. Patient characteristics were representative of community asthma, however, because documented characteristics of typical asthma patients include lack of spirometry, ${ }^{18}$ lack of receipt of guideline therapy, ${ }^{19}$ a high prevalence of "infectious asthma," 20 and prevalence of adult-onset asthma. ${ }^{10}$

So far, most asthma research has been designed and implemented from the perspective of asthma specialists. Less than $2 \%$ of community asthma patients are referred to specialists ${ }^{12}$ and, not surprisingly, these referred patients are disproportionately atopic. ${ }^{21}$ The current body of asthma research is heavily tilted toward short-term drug efficacy studies. ${ }^{13}$ To redress the balance, representative patient selection and practical clinical trial study design should be adopted in future asthma trials. Nationwide, more than two thirds of eligible asthma patients are not taking guideline therapy. ${ }^{19}$ One explanation for the discrepancy between recommendations and practice is lack of physician education regarding expert guidelines or perhaps lack of time to apply the guidelines. An alternative explanation for lack of guideline adoption is that physicians recognize that current asthma guidelines are insufficiently based on evidence from relevant generalizable studies of the patients they see in primary care. ${ }^{14}$

IVR data collection has intuitive appeal for reducing the burden of data acquisition in primary care-based randomized trials. In this study, significant amounts of follow-up data were not collected because some subjects with less than a high school education failed to use the IVR system consistently, although they did return for the follow-up visit. Future primary care-based studies aiming to recruit these subjects should consider alternative data collection methods, such as direct data gathering at follow-up visits. The small number of subjects with less than a high school education leads to uncertainty about generalizability; thus, further investigation is warranted into the utility of IVR data collection in practice-based research. For example, automated reminder phone calls were not used in this study but might have increased IVR response rate among those who failed to dial in regularly.
Because only two thirds of patient exit questionnaires were returned, interpretation of the responses is difficult because of possible selection bias. The responses do suggest that further primary care-based studies are feasible for most patients, who indicated they would have been willing to continue study participation but were not interested in using the IVR system more than once weekly.

In conclusion, our feasibility results indicate that primary care physicians were interested in and capable of performing an asthma RCT. We also found that it was possible to enroll primary care asthma patients using the broad eligibility criteria so important to generating relevant evidence on effective asthma management.

Co-investigators and staff enrolling patients: Erik Anderson, MD, Mary Nickel, Wausau, WI; Dennis Baumgartner, MD, Sarah Halsmer, Milwaukee, WI; Donald Bukstein, MD, Lottie Stenjem, Jon Senn, Madison, WI; Robert Feldman, MD, Chicago, IL; Cheri Olson, MD, Terri Pedace, LaCrosse, WI; Andrew Pasternak, MD, Reno, NV; John Sauret, MD, Buffalo, NY; Paul Smith, MD, Belleville, WI; Ellen Wiebe, MD, Ludek Podhradsky, Vancouver, BC, Canada. Other personnel and organizations: Pamela Wiesen, MBA, and Mary Beth Plane, PhD (Department of Family Medicine, University of Wisconsin Medical School and the Wisconsin Research Network) managed the Clinical Coordinating Center. The Investigational Drug Services, University of Wisconsin Hospitals and Clinics Department of Clinical Pharmacy managed the packaging, labeling, and distribution of study medication. Marlon Mundt, MS, Department of Family Medicine, University of Wisconsin Medical School, provided statistical consultant services. The interactive voice-response (IVR) telephone system was produced and managed by Healthcare Technology Systems (HTS) Inc., Madison WI.

\section{References}

1. Green LA. How can family practice and primary care practice-based research networks contribute to medical effectiveness research? in Hibbard H, Nutting PA, Grady ML, editors. Primary care research: theory and methods: conference proceedings. Rockville (MD): US Department of Health and Human Services, Public Health Service, Agency for Health Care Policy and Research; 1991.

2. Green LA, Hames CG Sr, Nutting PA. Potential of practice-based research networks: experience from ASPN. J Fam Pract 1994;38:400-6.

3. McBride P, Underbakke G, Plane MB, et al. Improving practice prevention systems in primary care: the Health Education and Research Trial (H.E.A.R.T.). J Fam Pract 2000;49:115-25.

4. Rost K, Nutting P, Smith JL, Elliot CE, Dickinson M. Managing depression as a chronic disease: a ran- 
domised trial of ongoing treatment in primary care. BMJ 2002;325:934.

5. Katzelnick DJ, Simon GE, Pearson SD, et al. Randomized trial of a depression management program in high utilizers of medical care. Arch Fam Med 2000;9:345-51.

6. Fleming MF, Barry KL, Manwell LB, Johnson K, London R. Brief physician advice for problem alcohol drinkers. A randomized controlled trial in community-based primary care practices. JAMA 1997; 277:1039-45.

7. Ryan JG, Velez FF, Schwartz ER, Patel AV. Family Medicine's contribution to industry-sponsored clinical research. Presented at the Annual Meeting of the North American Primary Care Research Group (NAPCRG); 2002 Nov 17-20; New Orleans, Louisiana, abstract CC4.

8. Hueston WJ. A comparison of albuterol and erythromycin for the treatment of acute bronchitis. J Fam Pract 1991;33:476-80.

9. Hueston WJ. Albuterol delivered by metered-dose inhaler to treat acute bronchitis. J Fam Pract 1994; 39:437-40.

10. Hahn DL, Beasley JW. Diagnosed and possible undiagnosed asthma: a Wisconsin Research Network (WReN) study. J Fam Pract 1994;38:373-9.

11. Arif AA, Delclos GL, Lee ES, Tortolero SR, Whitehead LW. Prevalence and risk factors of asthma and wheezing among US adults: an analysis of the NHANES III data. Eur Respir J 2003;21:827-33.

12. Forrest CB, Nutting PA, Starfield B, von Schrader S. Family physicians' referral decisions: results from the ASPN referral study. J Fam Pract 2002;51:215-22.

13. Aronson N, Lefevre F, Piper M, et al. Management of chronic asthma. Evidence Report/Technology Assessment Number 44. (Prepared by Blue Cross and Blue Shield Association Technology Evaluation
Center under contract no. 290-97-0015) AHRQ publication no. 01-E044. Rockville (MD): Agency for Healthcare Research and Quality; 2001.

14. Berg AO, Moy JG. Clinical guidelines and primary care: guidelines for the diagnosis and management of asthma. J Am Board Fam Pract 1992;5:629-34.

15. Tunis SR, Stryer DB, Clancy CM. Practical clinical trials. Increasing the value of clinical research for decision making in clinical and health policy. JAMA 2003;290:1624-32.

16. Expert Panel Report II. Guidelines for the diagnosis and management of asthma. Bethesda (MD): US Department of Health and Human Services, Public Health Service, National Institutes of Health, National Heart, Lung, and Blood Institute; 1997. Available from: http://www.nhlbi.nih.gov/guidelines/ asthma/asthgdln.htm

17. Dekker FW, Schrier AC, Sterk PJ, Dijkman JH. Validity of peak expiratory flow measurement in assessing reversibility of airflow obstruction. Thorax 1993;47:162-6.

18. Fried RA, Miller RS, Green LA, Sherrod P, Nutting PA. The use of objective measures of asthma severity in primary care: a report from ASPN. J Fam Pract 1995;41:139-43.

19. Fuhlbrigge A, Adams RJ, Guilbert TW, et al. The burden of asthma in the United States. Level and distribution are dependent on the interpretation of the National Asthma Education and Prevention Program guidelines. Am J Respir Crit Care Med 2002; 166:1044-9.

20. Hahn DL. Infectious asthma: a reemerging clinical entity? J Fam Pract 1995;41:153-7.

21. Li JT, Sheeler RD, Offord KP, Patel AM, Dupras DM. Consultation for asthma: results of a generalist survey. Ann Allergy Asthma Immunol 1999;83: 203-6. 